\title{
HISTORICAL EVOLUTIONARY INFORMATION IN THE TRADITIONAL LINNEAN HIERARCHY
}

BAMBACH*, Richard K., Dept. of Geological Sciences, Virginia Polytechnic Institute and State University, Blacksburg, VA 24061, U. S. A.; SEPKOSKI, J. John, Jr., Dept. of the Geophysical Sciences, University of Chicago, 5734 South Ellis Ave., Chicago, IL 60637, U. S. A.

The first two ranks above the species level in the traditional Linnean hierarchy - the genus and family - are species based: genera have been erected to unify groups of morphologically similar, closely related species and families have been erected to group genera recognized as closely related because of the shared morphologic characteristics of their species. Diversity patterns of traditional genera and families thus appear congruent with those of species in (a) the Recent (e. g., latitudinal gradients in many groups), (b) compilations of all marine taxa for the entire Phanerozoic (including the stage level), (c) comparisons through time within individual taxa (e. g., Foraminifera, Rugosa, Conodonta), and (d) simulation studies. Genera and families often have a more robust fossil record of diversity than species, especially for poorly sampled groups (e. g., echinoids), because of the range-through record of these polytypic taxa. Simulation studies indicate that paraphyly among traditionally defined taxa is not a fatal problem for diversity studies; in fact, when degradation of the quality of the fossil record is modelled, both diversity and rates of origination and extinction are better represented by including paraphyletic taxa than by restricting data to monophyletic clades. This result underscores the utility of traditional rank-based analyses of the history of diversity.

In contrast, the three higher ranks of the Linnean hierarchy - orders, classes and phyla - are defined and recognized by key character complexes assumed to be rooted deep in the developmental program and, therefore, considered to be of special significance. These taxa are unified on the basis of body plan and function, not species morphology. Even if paraphyletic, recognition of such taxa is useful because they represent different functional complexes that reflect biological organization and major evolutionary innovations, often with different ecological capacities. Phanerozoic diversity patterns of orders, classes and phyla are not congruent with those of lower taxa; the higher groups each increased rapidly in the early Paleozoic, during the explosive diversification of body plans in the Cambrian, and then remained stable or declined slightly after the Ordovician. The diversity history of orders superficially resembles that of lower taxa, but this is a result only of ordinal turnover among the Echinodermata coupled with ordinal radiation in the Chordata; it is not a highly damped signal derived from the diversity of species, genera, or families. Despite the stability of numbers among post-Ordovician Linnean higher taxa, the diversity of lower taxa within many of these Bauplan groups lluctuated widely, and these diversity patterns signal embedded ecologic information, such as differences in flexibility in filling or utilizing ecospace.

Phylogenetic analysis is vital for understanding the origins and genealogical structure of higher taxa. Only in such fashion can convergence and its implications for ecological constraints and/or opportunities be understood. But blind insistence on the use of monophyletic classifications in all studies would obscure some of the important information contained in traditional taxonomic groupings. The developmental modifications that characterize Linnean higher taxa (and traditionally separate them from their paraphyletic ancestral taxa) provide keys to understanding the role of shifting ecology in macroevolutionary success. 